\title{
ARTICLE The role of dopaminergic and serotonergic transmission in the processing of primary and monetary reward
}

\author{
Casper Schmidt ${ }^{1,2,3,4}$, Nikolina Skandali ${ }^{4,5}$, Carsten Gleesborg ${ }^{2,3}$, Timo L. Kvamme ${ }^{2,3,6}$, Hema Schmidt ${ }^{2,3}$, Kim Frisch ${ }^{2,3}$, \\ Arne Møller ${ }^{2,3}$ and Valerie Voon ${ }^{4,5}$
}

\begin{abstract}
Natural rewards such as erotic stimuli activate common neural pathways with monetary rewards. In human studies, the manipulation of dopamine and serotonin play an important role in the processing of monetary rewards with less understood on its role on erotic stimuli. In this study, we investigate the neuromodulatory effects of dopaminergic and serotonergic transmission in the processing of erotic versus monetary visual stimuli. We scanned one hundred and two $(N=102)$ healthy volunteers using functional magnetic resonance imaging while performing a modified version of the well-validated monetary incentive delay task consisting of erotic, monetary and neutral visual stimuli. We show a role for enhanced central dopamine and lowered central serotonin levels in increasing activity in the right caudate and left anterior insula during anticipation of erotic relative to monetary rewards in healthy controls. We further show differential activation in the anticipation of natural versus monetary rewards with the former associated with ventromesial and dorsomesial activity and the latter with dorsal cingulate, striatal and anterior insular activity. These findings are consistent with preclinical and clinical findings of a role for dopaminergic and serotonergic mechanisms in the processing of natural rewards. Our study provides further insights into the neural substrates underlying reward processing for natural primary erotic rewards and yields importance for the neurochemical systems of addictive disorders including gambling disorder.
\end{abstract}

Neuropsychopharmacology (2020) 45:1490-1497; https://doi.org/10.1038/s41386-020-0702-3

\section{INTRODUCTION}

What drives us towards natural rewards, such as food or sex? Characterizing neural substrates implicated in processing natural rewards is essential in understanding motivational and reward seeking processes, and pathological biases. Understanding how our behaviour may be biased, towards either drug or monetary rewards, or towards or away from natural food, sexual or social rewards, is relevant to the study of disorders of addiction. Here we focus on the substrates underlying central dopaminergic and serotonergic systems comparing primary natural sexual rewards and secondary monetary rewards in healthy humans.

Sexual behaviour has similar behavioural and neurofunctional underpinnings to other primary rewards, such as food. Sexual behaviour involves several neurophysiological systems, including endocrine, central nervous and peripheral mechanisms. The emergence of functional magnetic imaging has provided important neural insights. Sexual behaviour consists of motivation, consummation, and satiety phases. Sexual arousal comprises a cognitive components of appraisal and attention to sexual stimuli encoded by the orbitofrontal cortex, inferior temporal cortices and cerebellum, and an emotional component of sexual response as a primary reward encoded by the amygdala, insula and somatosensory cortices [1]. These studies focus on visual erotic stimuli, which reliably induces sexual motivation [2]. Central neurotransmitter systems, including dopamine (DA), serotonin (5HT) and noradrenaline, play a critical role in modulating sexual behaviour. In this study we assess the manipulation of dopamine and serotonin in the anticipation of monetary and sexual rewards.

\section{Serotonergic transmission and sexual behaviour}

Sexual dysfunction is a common side effect of selective serotonin reuptake inhibitors (SSRI) [3]. Sub-chronic paroxetine treatment of healthy male subjects decreased activation of the anterior cingulate cortex, including the subgenual, pregenual, and midcingulate cortices, ventral striatum and midbrain to visual erotic stimuli [4]. 5HT and DA have proposed opponent or functional, valence-dependent (aversive or appetitive), synergistic relationships [5]. Increasing prefrontal serotonergic levels appear to lower dopaminergic levels [6]. Administration in the same study sample of the serotonin-norepinephrine-dopamine reuptake inhibitor bupropion increased ventral striatal and midbrain activation attributed possibly to dopaminergic effects [2]. Most drugs of abuse exert acute effects on extracellular $5 \mathrm{HT}$

\footnotetext{
${ }^{1}$ Department of Communication and Psychology, Aalborg University, Rendsburggade 14, 9000 Aalborg, Denmark; ${ }^{2}$ Centre of Functionally Integrative Neuroscience, Aarhus

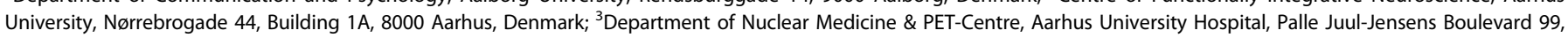

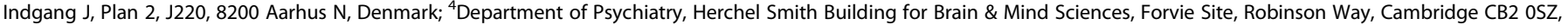

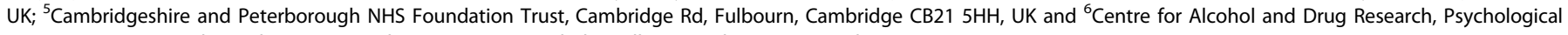
Institute, Business and Social Sciences, Aarhus University, Bartholins Allé 10, Aarhus C, Denmark

Correspondence: Valerie Voon (vv247@cam.ac.uk)

These authors contributed equally: Arne Møller, Valerie Voon
}

Received: 23 March 2020 Revised: 13 April 2020 Accepted: 20 April 2020

Published online: 11 May 2020 
activity: stimulant drugs, heroin and alcohol increase rodent serotonergic transmission in brain areas implicated in reward processing $[7,8]$.

The role of dopaminergic transmission in the processing of erotic rewards

Dopamine plays a critical role in reward processing and reinforcement learning with midbrain dopaminergic neurons encoding reward prediction errors, the difference between expected and experienced rewards [9] and the incentive salience of reward-related stimuli [10]. L-dopa administration and DA D2/3 receptor agonists are associated with new onset gambling disorder and compulsive sexual behaviours in patients with Parkinson's disease $[11,12]$.

Drugs of abuse and natural rewards share a common neural pathway, the mesolimbic dopamine system, in both a similar and distinct way between sexual rewards and effects of drugs of abuse [13]. Dopamine mediates cue-induced craving, cognitive control and behavioural inflexibility that characterize addiction disorders, and increases in dopamine transmission underlie the reinforcing effects of drugs of abuse [14]. Sexual experience followed by abstinence shows cross-sensitization with drug rewards in rodents by inducing neuroplasticity mediated by the transcriptional factor $\triangle$ FosB via $D_{1}$ receptor activation in the nucleus accumbens (NAcc) [15]. Dopaminergic receptor antagonism of mesolimbic/mesocortical reward systems is presumed to be related to sexual dysfunction observed in up to $70 \%$ of treated schizophrenia patients [16]. However gambling and hypersexuality have been reported with aripiprazole which has partial D3 receptor agonist effects [17].

\section{Common neural substrates between sexual and monetary} rewards?

Sexual and monetary rewards appear to be mediated by a common brain network, which includes the ventromedial prefrontal cortex (vmPFC), amygdala, ventral striatum, mediodorsal thalamus and anterior insula [18]. One of the most wellestablished tasks to dissociate anticipation and receipt of reward stimuli is the monetary incentive delay (MID) task [19], which has been extensively used in functional magnetic resonance imaging (fMRI) studies of healthy controls and addiction disorders. The ventral striatum is implicated in reward anticipation for both primary and secondary rewards. Pathological gamblers showed reduced ventral striatal activation to anticipated erotic compared to monetary stimuli in an adapted MID task accompanied by reduced behavioural motivation towards erotic stimuli [20]. In contrast, subjects with compulsive sexual behaviour (CSB) or problematic pornography use showed greater ventral striatal activation to erotic anticipation in this same adapted MID task compared with healthy volunteers, an effect not observed for monetary rewards [21]. Here we seek to understand the neurochemical substrates in healthy controls to monetary and sexual reward processing underlying these observations in pathological groups.

Study aim

We address the role of dopaminergic and serotonergic manipulation on the processing of sexual as compared to monetary rewards in a large sample of healthy volunteers. We increased central DA levels with L-dopa (the precursor of DA) and carbidopa (an inhibitor of peripheral metabolism of L-dopa) and we decreased central $5 \mathrm{HT}$ levels with acute tryptophan depletion (ATD) [22]. Participants were scanned with fMRI while performing a modified MID task with both monetary and visual erotic rewards. We predicted that increased DA levels would enhance striatal activity to monetary rewards and that increased DA and decreased $5 \mathrm{HT}$ levels would enhance striatal activity to the anticipation of sexual rewards.

\section{PATIENTS AND METHODS}

Recruitment

One hundred and two healthy volunteers, aged $18-50$ years old, were recruited using written, electronic and social media advertisements. Given the nature of the sexually explicit material presented, all participants were male and heterosexual. Participants were included if they were medication-free, had no active psychiatric illnesses or substance use disorder (Mini International Neuropsychiatric Inventory assessed by a psychologist) and were MRI eligible. Participants completed the Profile of Mood States [23] prior and after medication administration, the Beck Depression Inventory (BDI) [24], the State-Trait Anxiety Inventory (STAI) [25], a digit span test from the Wechsler Adult Intelligence Scale [26], and the Danish version of the National Adult Reading Test (DART) [27] as a proxy of intelligence. The study was approved by the Middle Jutland Scientific Ethical Committee and all participants provided written and informed consent.

Pharmacological manipulation/medication

For the L-dopa preparation, participants received $100 \mathrm{mg}$ L-dopa (Sinemet), the precursor of dopamine, and $25 \mathrm{mg}$ carbidopa, an inhibitor of aromatic amino acid decarboxylase. Participants were scanned $\sim 1 \mathrm{~h}$ after drug ingestion to ensure optimal DA plasma concentration [28]. For the ATD, participants received a liquid amino acid load without tryptophan but with other large neutral amino acids, as per previous studies [29]. Participants were scanned $2 \mathrm{~h}$ post administration to ensure successfully lowered tryptophan levels [22]. Further details on the experimental procedure can be found in Supplementary Material and methods.

Study groups

Participants were divided into four groups consisting of (1) L-dopa treatment alone, (2) ATD treatment alone, (3) L-dopa and ATD, and 4. controls, receiving no pharmacological manipulation.

The MID task

Participants completed a modified MID task consisting of monetary, erotic and neutral reward outcomes (Fig. 1), previously described in Sescousse et al. [20]. Participants react to a target stimulus presented after an incentive cue to obtain the anticipated reward. Each trial consisted of the reward cue, anticipation, discrimination, and outcome. The cue indicated the type of reward. Anticipation was represented using a fixation cross (1500-4500 ms). A visual discrimination task was then performed within $1000 \mathrm{~ms}$ (with reaction time stair-cased to outcome: correct response subtracted $50 \mathrm{~ms}$ from the allowed response time, and incorrect response added $50 \mathrm{~ms}$ ); successful timely correct responses led to the reward outcome (i.e., a sexually explicit image or a monetary reward) and slow or incorrect responses led to reward omission. No reward (neutral outcome) trials were represented using scrambled pictures of sexually explicit ones to adjust for visual processing, including image size, color and complexity. The erotic stimuli for the task were chosen from a validated stimulus set [21] (24 pictures of undressed females), and monetary rewards was represented by a safe with the amount indicated (in Danish kroner) along with the cumulative winnings. Ratings of the erotic images for liking (pleasantness) and wanting (sexual arousal) were recorded at the end of task on a scale from one to ten to account for variability in sexual preferences and control for desensitization to the sexually explicit material. Participants also performed additional cognitive tests (reported elsewhere).

Data acquisition and processing

MRI data were acquired with a Siemens Skyra 3T scanner with a 32-channel head coil at the Center of Functionally Integrative Neuroscience at the Danish Neuroscience Center in Aarhus Denmark. Sequences comprised of a field-map acquisition 


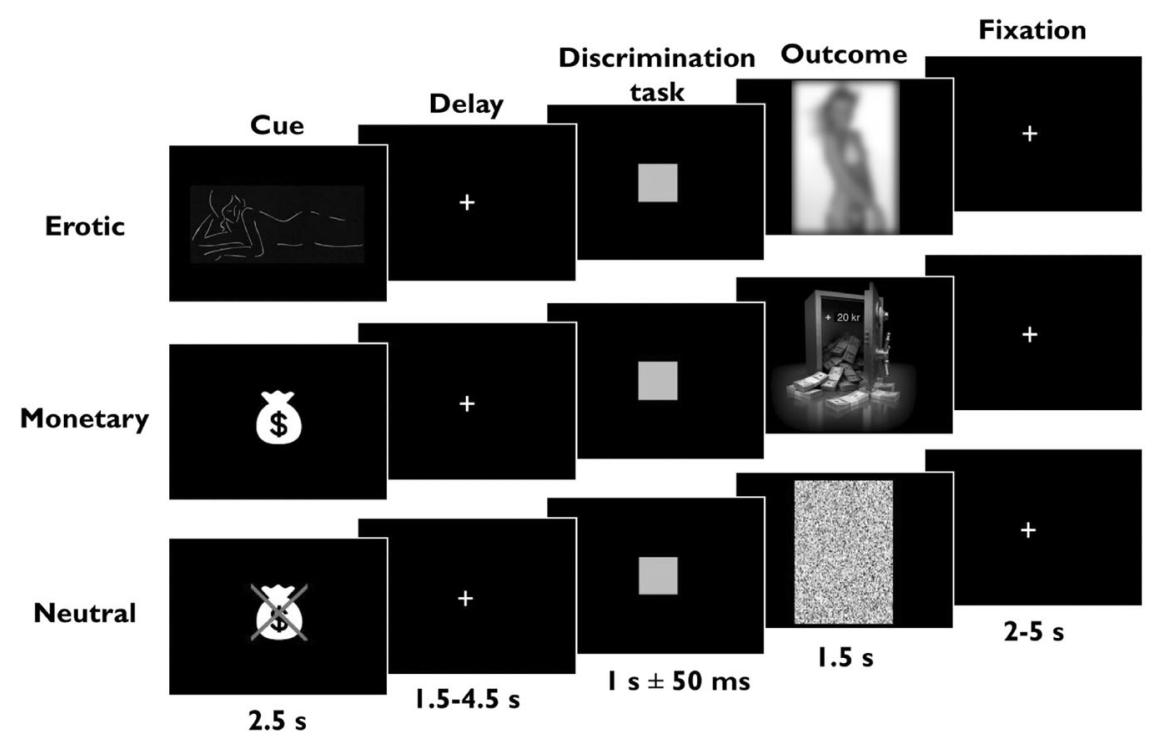

Fig. 1 The monetary incentive delay task (MID). Participants were first shown a cue indicating the reward type. The cue was then replaced by a fixation cross indicating the anticipation phase and then the discrimination task, where they had to make a correct response (button press to a symbol within $1 \mathrm{~s}$ ) to obtain the reward. In correct and rewarded trials, subjects were shown either a safe with information about the amount won and cumulative winnings, or a sexually explicit picture of a female.

(multi-band, single-echo) and a structural T1-weighted MP2RAGE sequence. An echo-planar imaging (EPI) sequence was acquired of 1200 volumes (repetition time (TR): $1660 \mathrm{~ms}$, echo time (TE): $30 \mathrm{~ms}$, flip angle: $75^{\circ}$, acquisition matrix: $106 \times 106 \times 72$ ).

Preprocessing of the functional EPI images was performed with Statistical Parametric Mapping, 12th edition [30]. Converted NIFTI images were slice time corrected to the center of each TR period, realigned, and co-registered to the same EPI image from SPM using the OldNormalise method (based on three comparisons of registration and normalization, including T1, field-map and EPI images, as the latter yielded higher spatial resolution in orbitoand medial frontal regions, when compared with field-map and segmentation-based methods) [31]. Images were smoothed with a full width at half maximum kernel of $6 \mathrm{~mm}^{3}$. Realignment parameters were included as a covariate. Second-level analyses were performed dividing participants into groups based on blinded IDs and computed separately for anticipation and outcome phases in a full factorial design specification, with a between-subjects factor of drug (assuming independence and unequal variance), and a within-subjects factor of type of reward (assuming non-independence and equal variance). Tests were employed for main effects of drug and reward for the separate phases of reward anticipation and outcome for all four groups. If main effects were found, drug by reward interaction effects were analyzed with two-sample t-tests. Reward types were compared with neutral as a control (i.e., monetary anticipation vs. neutral anticipation; erotic anticipation vs. neutral anticipation). All analyses were reported at whole-brain family-wise error $p<0.05$ cluster corrected (with cluster defining threshold of $p<0.001$ uncorrected). Region-of-interest (ROI) analyses focused on the ventral striatum and were conducted with small-volumecorrection of $5 \mathrm{~mm}$ with significance assigned at a threshold of family-wise error $p<0.05$.

Statistical analysis of behavioural outcomes

Participant demographics and trait questionnaire scores were assessed for normality of distribution and outliers and compared between groups with two-tailed $t$ tests. No outliers were noted. Due to the technical errors, reaction time data for four participants were unavailable. We examined RT differences across erotic and monetary versus neutral targets using an improved-scoring algorithm by Greenwald et al. [32]. This algorithm reduces biases due to variability of RTs by standardizing differences in response latencies and dividing an individual's difference in RTs by a personalized standard deviation of these differences. We compared drug by reward RT differences using a two-way analysis of variance (ANOVA). For trait personality and mood state ratings, we performed two-way ANOVAs. For significant main effects, posthoc $t$ tests were Bonferroni corrected for multiple comparisons. Effect sizes were reported using partial Eta Squared (pn2). Statistical analyses were performed using the statistical software $\mathrm{R}$ (version 3.6.3).

\section{RESULTS}

Behavioural results

Demographics and questionnaire results are presented in Table 1 with no significant differences noted. Participants also did not differ in measures of alcohol or pornography craving or impulsivity. Across all groups, we show faster RTs to monetary compared to erotic cues $\left(F(1,188)=9.36, p=0.003, p \eta^{2}=0.047\right)$ with no medication effect. Subsequent pairwise $t$ tests revealed that only the L-dopa group did not show a significant difference $\left(t(24)=1.88, p=0.29, p^{\text {uncorrected }}=0.07\right)$, whereas ATD $(t(22)=$ 2.74, $p=0.048), \mathrm{ATD}+\mathrm{LD}(\mathrm{t}(22)=2.89, p=0.033)$, and the HVcontrol groups $(t(26)=2.73, p=0.04)$ all reached significance.

Reward anticipation neural activity

The main effect of medication during reward anticipation revealed greater activity in the drug groups in the right parahippocampus, ventral posterior cingulate cortex (vPCC), and right primary visual cortex relative to placebo (for full results overview, see Table S1 in Supplementary Material). The main effect of reward revealed differences across erotic and monetary reward anticipation. Anticipation of erotic-neutral stimuli was associated with increased mesial-prefrontal activity in the vmPFC, dorsomedial prefrontal cortex (dmPFC) and VPCC, whereas monetary-neutral anticipation was associated with increased bilateral ventral striatum, caudate, putamen, anterior insula, and $\mathrm{dACC}$ and right dIPFC (Fig. 2).

We then showed that erotic-neutral anticipation in drug groups versus monetary-neutral anticipation in placebo had greater 
Table 1. Demographic characteristics and questionnaire results. Mean \pm standard deviation (SD).

\begin{tabular}{|c|c|c|c|c|c|c|}
\hline Variable & L-dopa & ATD & L-dopa + ATD & $\mathrm{HV}$ & $F$ & $P$ \\
\hline DART & $27.9(7.9)$ & $31.1(6.3)$ & $33.0(3.7)$ & $27.9(6.6)$ & 2.82 & 0.04 \\
\hline $\mathrm{BDI}$ & $5.4(4.9)$ & $5.4(3.7)$ & $6.1(3.6)$ & $7.2(8.5)$ & 0.53 & 0.66 \\
\hline STAI & $60.7(12.6)$ & $67.7(11.3)$ & $68.2(15.3)$ & $66.8(17.4)$ & 1.49 & 0.22 \\
\hline Erotic RT bias & $0.3(0.3)$ & $0.3(0.3)$ & $0.4(0.3)$ & $0.4(0.3)$ & 0.94 & 0.43 \\
\hline Monetary RT bias & $0.5(0.4)$ & $0.5(0.3)$ & $0.6(0.3)$ & $0.6(0.4)$ & 0.52 & 0.67 \\
\hline
\end{tabular}

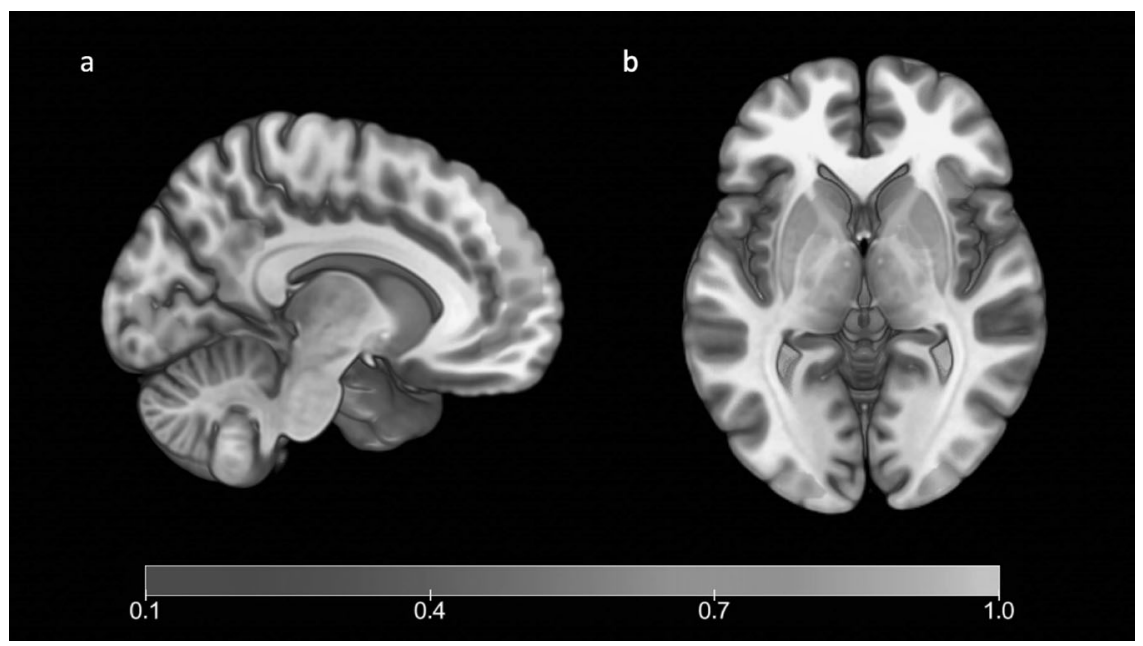

Fig. 2 Main effect of reward anticipation. Main effect of reward with (a) greater erotic relative to monetary anticipation activity in ventromedial and dorsomedial prefrontal cortices, and ventral posterior cingulate cortex, and (b) greater monetary relative to erotic anticipation activity in bilateral areas of ventral striatum, caudate, putamen and anterior insula, and the dorsal anterior cingulate cortex and the right dorsolateral prefrontal cortex. The image was thresholded at the whole-brain level of $p=0.001$ uncorrected, and made in MRIcroGL (ver. 1.150909) by manually extracting cluster from Statistical Parametric Mapping and adding an overlay illustrating signal intensity.

activity in right caudate, left anterior insula and left cerebellum (Fig. 3). Post-hoc analyses showed no significant differences among the three different medication groups. There were no significant differences in the opposite contrast.

\section{Reward outcome neural activity}

The main effect of drug showed that drug groups relative to placebo had greater activity in the right vPCC, left parahippocampus, and medial cerebellum (Figure S1 in Supplementary Material). Further ROI analyses for the bilateral ventral striatum revealed increased activity in drug groups relative to placebo.

The main effect of reward outcome showed that erotic-monetary outcomes had greater activity in mesialprefrontal regions such as the vmPFC, subgenual cingulate cortex, bilateral OFC, amygdala and fusiform face areas (Fig. 4). In contrast, monetary-erotic outcomes showed greater activity in $\mathrm{dACC}$, and anterior PFC. There was no interaction between the drug type and reward type.

\section{DISCUSSION}

We acutely increased central dopaminergic transmission with the dopamine precursor L-dopa and decreased central serotonergic transmission with ATD in a large group of non-clinical healthy volunteers. Participants underwent fMRI scanning while performing a well-validated version of the MID task consisting of monetary, erotic and neutral stimuli. We show activation of distinct neural systems for erotic and monetary rewards during anticipation and outcome stages. We additionally show activation of dissociable neural areas by altered dopaminergic and serotonergic levels compared to the placebo group.

Dissociable neural areas encoding erotic versus monetary rewards Erotic stimuli during both anticipation and outcome stages activated mesial-prefrontal areas such as vmPFC and dmPFC, whereas monetary rewards activated dorsal cingulate areas. The vmPFC is implicated in outcome representation and associative learning relevant to outcome tracking of goal-directed control [33], whereas the dorsal cingulate is implicated in salience, tracking of prediction errors and conflict monitoring [34]. The two brain areas have been differentially involved in foraging versus decisions regarding traditional economic choices [35] with proposed distinct involvement of vmPFC and dACC for current choice and best long-term option respectively in sequential multiple-stage decisions [36]. It is plausible that the distinct neural activation in our study represents potentially different decision 
processes for natural (sexual) versus secondary (monetary) rewards; although our task is not a sequential decision-making task, it may well be that trials with monetary rewards tracked longer term options, which included showing the cumulative

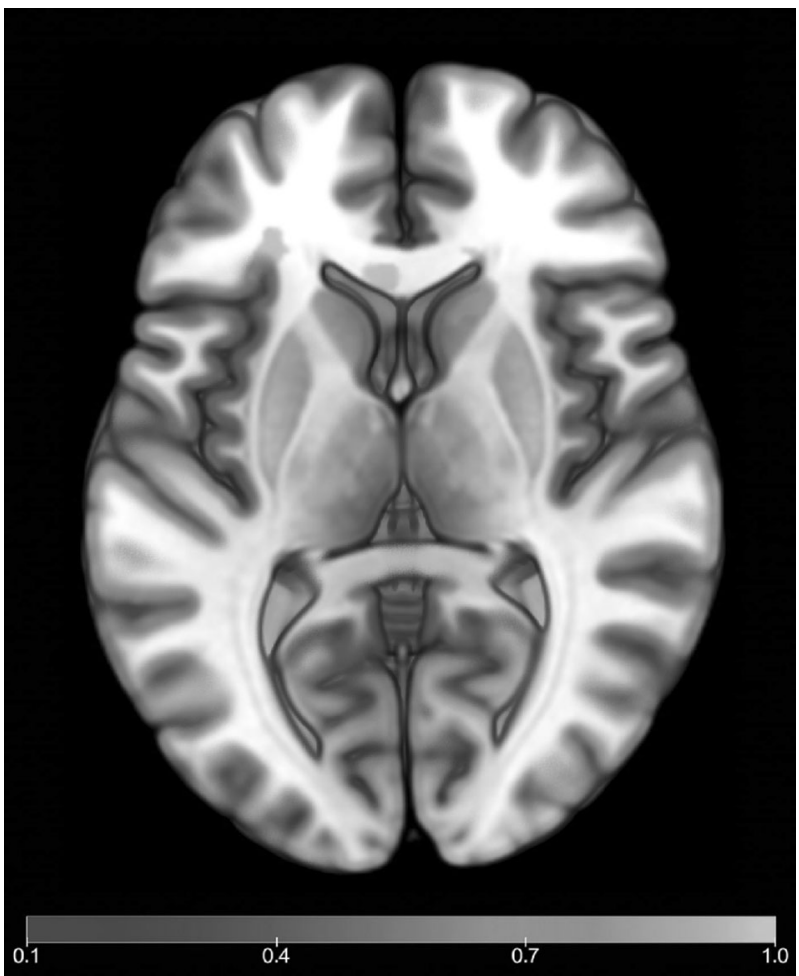

Fig. 3 Drug by reward interaction effect with increased erotic versus monetary anticipation in medicated groups compared to the control group in the right caudate and left anterior insula. Image thresholded at the whole-brain level of $p=0.001$ uncorrected, and made in MRIcroGL (ver. 1.150909) by manually extracting cluster from Statistical Parametric Mapping and adding overlays illustrating signal intensity. earnings, elicited the outcomes of decisions to be used as priors for the next trial presenting with monetary rewards.

We also show enhanced striatal and anterior insula activation during anticipation of monetary rewards which may also represent a greater preference in healthy controls. Primary rewards including food and erotic rewards were previously shown to be associated with anterior insular activity compared to monetary rewards [20]. The anticipation stage for erotic rewards in our study possibly activates the cognitive component as described in the neurophenomenological model already detailed, i.e., attention and appraisal to the visual sexual stimuli; this includes the activation of prefrontal areas but not striatal and insular areas, which are associated along with amygdala with the emotional component of the model. Indeed, during the reward outcome stage, erotic stimuli increased amygdala activation. In addition, in our study we applied visual erotic stimuli; although this reliably induces sexual motivation and "wanting", it is distinct from genital stimulation [37], which is required for entering the consummatory plateau of the sexual response cycle and activates insula and amygdala [2].

Our findings also highlight differences during the reward outcome stage with greater OFC activity for erotic versus monetary reward outcomes. A previous study using a similar adapted MID task showed enhanced activity in posterior OFC to erotic versus monetary reward outcomes, a finding that was greater in non-treatment-active gamblers to monetary outcomes [20]. This difference may represent dissociate tracking of different reward types differentiating between a primary natural reinforcer and a secondary conditioned reinforcer, which may represent distinct evolutionary processes [20]. Indeed, rodent studies suggest that natural sweet taste reward and drug of abuse (heroin) are both represented in different neuronal populations within the OFC [38]. In addition, the OFC is highly interconnected with the basolateral amygdala encoding expected outcomes and coordinating goal-directed behaviour [39]. Alternatively, monetary anticipation and tracking of monetary outcomes may involve more discrete representations of reward value and prediction errors which might be more closely associated with dorsal cingulate and striatal regions. The OFC is also implicated in behavioural disinhibition [40] and the lateral OFC particularly in inhibitory failures [41], which may contribute to impairments in inhibitory control in the context of erotic imagery.

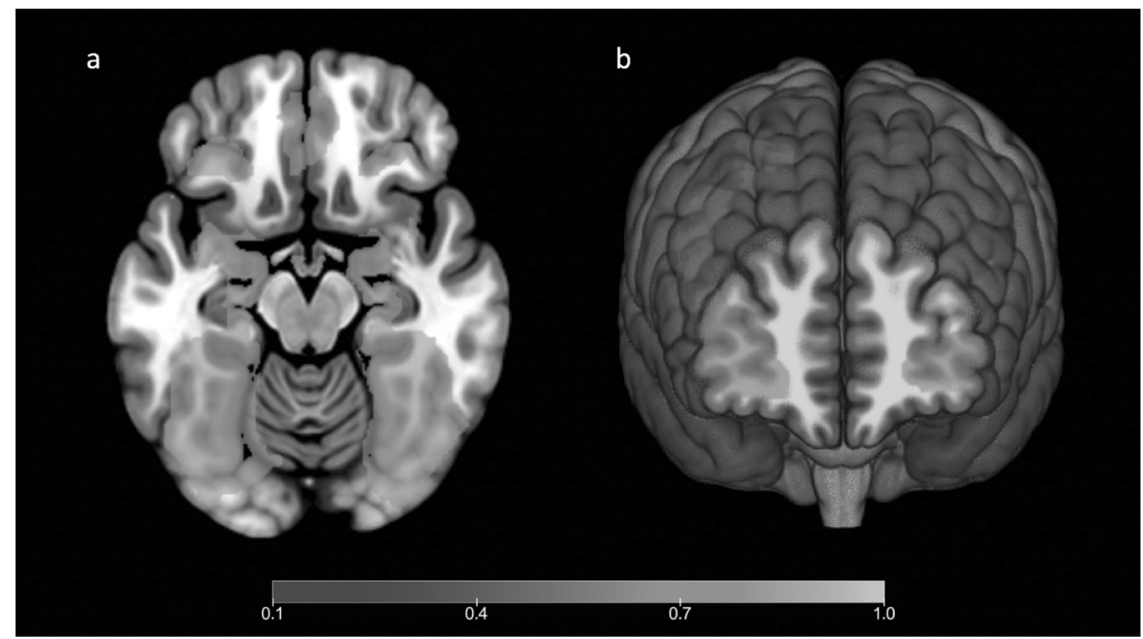

Fig. 4 Main effect of reward outcome. Main effect of Reward with (a) greater erotic relative to monetary outcome activity in ventromedial, subgenual cingulate, and bilateral orbitofrontal cortices, and bilateral amygdala and fusiform face area, and (b) greater monetary relative to erotic anticipation activity in bilateral anterior prefrontal and dorsal anterior cingulate cortices. The image was thresholded at the whole-brain level of $p=0.001$ uncorrected, and made in MRIcroGL (ver. 1.150909) by manually extracting cluster from Statistical Parametric Mapping and adding an overlay illustrating signal intensity. 
Pharmacological manipulation of erotic versus monetary rewards We show that increased DA and decreased 5HT tone increased activation of the right caudate and left anterior insula during anticipation of erotic relative to monetary rewards in healthy controls (Fig. 3). We have very recently shown that treatmentactive gambling disorder subjects relative to healthy volunteers have greater ventral striatal activity to erotic versus monetary reward anticipation (work currently submitted). Our findings in this healthy control group suggest that one plausible mechanism for our observations in gambling disorder subjects may relate to enhanced dopaminergic and lowered serotonergic tone. These findings converge with preclinical and clinical studies on the role of dopaminergic and serotonergic effects on sexual reward.

These findings converge with and also shed light on the literature on CSB suggesting that differential mechanisms may lead to similar behavioural profiles. Dopamine agonists have been associated with CSB in Parkinson's disease [42] with greater sexual drive associated with dopaminergic drugs correlating with greater striatal, cingulate and OFC activity to sexual imagery [43]. Our findings are consistent with these observations of dopamineagonist-related CSB. However, PD is also associated with neurodegeneration across monoaminergic cell bodies including the serotonergic system; patients that develop CSB represent a specific subgroup, which might also have greater baseline impairments in underlying serotonergic function. In the general population, CSB is associated with enhanced ventral striatal activity to visual sexual cues [44] associated with greater early attentional bias [45] correlating with greater choice preference and approach behaviours towards sexually conditioned stimuli [45], suggesting potential overlaps between CSB and disorders of addiction. CSB subjects also show greater ventral striatal activity during the anticipation of sexual imagery relative to monetary rewards as tested using a similarly modified version of the MID task [21]. Our findings suggest a possible role for underlying hyperactive dopaminergic or lower central serotonin tone in CSB. Further studies in this population with pharmacological manipulation may shed more light on causal mechanisms.

Preclinical neuroimaging studies in humans have suggested a link between antidepressant treatment and decreased sexual function thus lending support to the idea that increased 5HT might decrease sexual motivation [46]. We show that depleting central $5 \mathrm{HT}$ levels may increase neural properties of anticipation of sexual rewards. Given the suggested modulatory role of serotonergic transmission on the dopaminergic system $[5,6]$, the observed effects may be mediated through dopaminergic transmission. Innate natural rewards such as sweet taste appear to override cocaine including in drug-sensitized rodents [47] mediated possibly by the endocannabinoid system [48], which in turn is correlated with increased DA release in the NAcc [49]. Indeed, higher extracellular dopaminergic levels were shown to result in increased "wanting" for sweet rewards [50]. Similarly, we show that increases in DA and lowering $5 \mathrm{HT}$ enhances caudate activity specifically to erotic relative to monetary anticipation in healthy volunteers.

We did not show a specific effect of drug on reward outcomes. Previous studies using similar adapted MID tasks in CSB demonstrated only differences in the anticipation and not outcome phase in keeping with the concept of higher 'wanting' rather than 'liking' of erotic outcomes [21]. We did show increased ventral striatal activity in the drug groups to reward outcomes. We applied three different manipulations consisting of increased dopaminergic, lowered serotonergic, and a combination of increased dopaminergic and lowered serotonergic transmission and compared these to placebo. We did not find any difference between the three different types of neuromodulation, but we found that all three manipulations increased activity in bilateral ventral striatal areas during reward outcomes characterized by dense dopaminergic innervation [51]. The ventral striatum is one of the neural areas within the reward processing circuit which along with the orbitofrontal cortex, amygdala and medial prefrontal cortex, and it is suggested to mediate reward prediction errors which drive reinforcement learning [9]. Both primary [52] and secondary rewards [53] activate ventral striatum; indeed, in our study we did not observe any role for the type of reward on activation of this neural area. This lack of ventral striatal difference in the anticipation of reward types might reflect the lack of differential preference in healthy controls, whereas in CSB subjects, sexual reward preference appears to play a role in greater ventral striatal activity [54]. A role for serotonin mediating an inhibitory control of the prefrontal cortex over reward signals encoded by dopaminergic neurons in the NAcc has been suggested $[6,55]$. Negative interactions between the NAcc and anteroventral PFC, which is suggested as a neural correlate of prefrontal inhibitory control over reward signals in the NAcc [56], were increased with the SSRI paroxetine during processing of erotic rewards [4]. Thus, our findings align with previous findings of potential opponent interaction between dopaminergic and serotonergic transmission and show this interaction may also underlie the processing of erotic rewards.

This study is not without limitations. The study is focused on healthy controls; such pharmacological manipulations in patient groups using this fMRI task might allow for more causative interpretations. We did not observe any differences between interventions. These specific manipulations may not be sensitive to reward anticipation and outcome but might reveal differences with other cognitive processes or within patient groups. Levodopa administration and tryptophan depletion are also fairly nonspecific interventions; more specific pharmacological manipulations on specific dopamine or serotonin receptor subtypes might be indicated to reveal differential effects.

Together, our findings demonstrate dissociable effects of altered central dopamine and serotonin levels in the processing of erotic versus monetary rewards in a non-clinical healthy population. Our findings provide further insights into the neuromodulatory systems of reward processing. These findings also have important clinical implications. Our findings suggest that multiple possibly interactive mechanisms may lead to CSB. The management of dopaminergic medication-induced CSB in Parkinson's disease beyond decreasing dose or changing medications can be very difficult to treat. The capacity to differentially modulate reward subtypes, for instance by enhancing natural rewards in alcohol or drug addiction or gambling disorders might shift the myopic bias away from pathological cues to natural rewards. Serotonin-specific reuptake inhibitors have been shown to improve gambling disorder [57]; one plausible mechanism by which this might occur might be related to a shift from pathological towards natural rewards. The role of serotonergic mechanisms in CSB may be complex as a small case series of CSB subjects treated with serotonin specific reuptake inhibitors show an initial decrease in compulsive sexual behaviours but a longer term increase in new sexual behaviours [58]. Further pharmacological studies with specific 5HT receptor profiles or dopamine antagonist profiles are indicated in CSB. Hypoactive sexual desire in women and sexual dysfunction related to chronic antidepressants and antipsychotics is a highly relevant clinical problem. Flibanserin a $5 \mathrm{HT} 1 \mathrm{~A}$ agonist and $5 \mathrm{HT} 2 \mathrm{~A}$ antagonist shows efficacy in the treatment of hypoactive sexual desire in women [59]. The dopamine-agonist apomorphine has also shown efficacy in hypoactive sexual desire in women [60]. Bupropion, a noradrenergic and dopaminergic reuptake inhibitor shows efficacy in the management of serotonin reuptake inhibitor sexual dysfunction in depression [61] and methadone-treated patients [62] and demonstrates differential neural activity to erotic stimuli relative to paroxetine [4]. Further studies assessing the role of pharmacological manipulation in pathological disorders along with a more fine-grained understanding of the specific dopamine or serotonin receptor subtype, and the role of other neurotransmitters are indicated. 


\section{FUNDING AND DISCLOSURE}

The study's first author received funding from the Augustinus Foundation and the Institute of Clinical Medicine at Aarhus University. VV is supported by a Medical Research Council Senior Clinical Fellowship (MR/P008747/1). The authors declare no competing interests.

\section{AUTHOR CONTRIBUTIONS}

Each author's contributions are listed in the author order using initials to indicate their contributions to study design (1); or the acquisition (2), analysis (3), or interpretation of data for the work (4); and in drafting the work or revising it critically for important intellectual content (5); and final approval of the version to be published (6): CS: 1-6 NS: 4-6 CG: 1,3,6 TK; 2-3,6 HS: 2-3,6 KF: 2,6, AM: 1-2,4-6 VV: 1-6.

\section{ADDITIONAL INFORMATION}

Supplementary Information accompanies this paper at (https://doi.org/10.1038/ s41386-020-0702-3).

Publisher's note Springer Nature remains neutral with regard to jurisdictional claims in published maps and institutional affiliations.

\section{REFERENCES}

1. Stoléru S, Fonteille V, Cornélis C, Joyal C, Moulier V. Functional neuroimaging studies of sexual arousal and orgasm in healthy men and women: a review and meta-analysis. Neurosci Biobehav Rev. 2012;36:1481-509.

2. Graf H, Malejko K, Metzger C, Walter M, Grön G, Abler B. Serotonergic, dopaminergic, and noradrenergic modulation of erotic stimulus processing in the male human brain. J Clin Med. 2019;8:363.

3. Graf H, Walter M, Metzger CD, Abler B. Antidepressant-related sexual dysfunction - perspectives from neuroimaging. Pharmacol Biochem Behav. 2014;121:138-45.

4. Abler B, Seeringer A, Hartmann A, Grön G, Metzger C, Walter M, et al. Neural correlates of antidepressant-related sexual dysfunction: a placebo-controlled fmri study on healthy males under subchronic paroxetine and bupropion. Neuropsychopharmacology. 2011;36:1837-47.

5. Boureau YL, Dayan P. Opponency revisited: competition and cooperation between dopamine and serotonin. Neuropsychopharmacology. 2011;36:74-97.

6. Seo D, Patrick CJ, Kennealy PJ. Role of serotonin and dopamine system interactions in the neurobiology of impulsive aggression and its comorbidity with other clinical disorders. Aggress Violent Behav. 2008;13:383-95.

7. Ikeda R, Igari $Y$, Fuchigami $Y$, Wada M, Kuroda N, Nakashima K. Pharmacodynamic interactions between MDMA and concomitants in MDMA tablets on extracellular dopamine and serotonin in the rat brain. Eur J Pharm. 2011;660:318-25.

8. Parsons LH, Justice JB Jr. Serotonin and dopamine sensitization in the nucleus accumbens, ventral tegmental area, and dorsal raphe nucleus following repeated cocaine administration. J Neurochem. 1993;61:1611-9.

9. Schultz W, Dayan P, Montague PR. A neural substrate of prediction and reward. Science. 1997;275:1593-9.

10. Berridge KC, Robinson TE. What is the role of dopamine in reward: hedonic impact, reward learning, or incentive salience? Brain Res Rev. 1998;28:309-69.

11. Weintraub D, Koester J, Potenza MN, Siderowf AD, Stacy M, Voon V, et al. Impulse control disorders in Parkinson disease: a cross-sectional study of 3090 patients. Arch Neurol. 2010;67:589-95.

12. Voon V, Hassan K, Zurowski M, De Souza M, Thomsen T, Fox S, et al. Prevalence of repetitive and reward-seeking behaviors in Parkinson disease. Neurology. 2006;67:1254-7.

13. Frohmader KS, Wiskerke J, Wise RA, Lehman MN, Coolen LM. Methamphetamine acts on subpopulations of neurons regulating sexual behavior in male rats. Neuroscience. 2010;166:771-84.

14. Volkow ND, Fowler JS, Wang GJ, Swanson JM, Telang F. Dopamine in drug abuse and addiction: Results of imaging studies and treatment implications. Arch Neurol. 2007;64:1575-9.

15. Pitchers KK, Vialou V, Nestler EJ, Laviolette SR, Lehman MN, Coolen LM. Natural and drug rewards act on common neural plasticity mechanisms with $\triangle \mathrm{FosB}$ as a key mediator. J Neurosci. 2013;33:3434-42.

16. Dossenbach M, Dyachkova Y, Pirildar S, Anders M, Khalil A, Araszkiewicz A, et al. Effects of atypical and typical antipsychotic treatments on sexual function in patients with schizophrenia: 12-month results from the Intercontinental Schizophrenia Outpatient Health Outcomes (IC-SOHO) study. Eur Psychiatry. 2006;21:251-8.
17. Moore TJ, Glenmullen J, Mattison DR. Reports of pathological gambling, hypersexuality, and compulsive shopping associated with dopamine receptor agonist drugs. JAMA Intern Med. 2014;174:1930-3.

18. Sescousse G, Caldú $X$, Segura B, Dreher JC. Processing of primary and secondary rewards: a quantitative meta-analysis and review of human functional neuroimaging studies. Neurosci Biobehav Rev. 2013;37:681-96.

19. Knutson B, Greer SM. Review. Anticipatory affect: Neural correlates and consequences for choice. Philos Trans R Soc B Biol Sci. 2008;363:3771-86.

20. Sescousse G, Barbalat G, Domenech P, Dreher JC. Imbalance in the sensitivity to different types of rewards in pathological gambling. Brain. 2013;136:2527-38.

21. Gola M, Wordecha M, Sescousse G, Lew-Starowicz M, Kossowski B, Wypych M, et al. Can pornography be addictive? An fMRI study of men seeking treatment for problematic pornography use. Neuropsychopharmacology. 2017;42:2021-31.

22. Young S. Acute tryptophan depletion in humans: a review of theoretical, practical and ethical aspects. J Psychiatry Neurosci. 2013;38:294-305.

23. Grove JR, Prapavessis $H$. Preliminary evidence for the reliability and validity of an abbreviated profile of mood states. Int J Sport Psychol. 1992;23:93-109.

24. Dozois DJA. Beck Depression Inventory-II. Corsini Encycl Psychol. 2010;78:490-8.

25. Spielberger CD. State-trait anxiety inventory for adults. 1983.

26. Wechsler D. Wechsler adult intelligence scale-Fourth Edition (WAIS-IV). San Antonio, TX: Psychol Corp; 2014.

27. Nelson HE, Willison J. National Adult Reading Test (NART) NFER. vol. UK: NFER-N; 1991.

28. Rutledge RB, Skandali N, Dayan P, Dolan RJ. Dopaminergic modulation of decision making and subjective well-being. J Neurosci. 2015;35:9811-22.

29. Worbe Y, Palminteri S, Savulich G, Daw ND, Fernandez-Egea E, Robbins TW, et al. Valence-dependent influence of serotonin depletion on model-based choice strategy. Mol Psychiatry. 2016;21:624-9.

30. Penny WD, Friston KJ, Ashburner JT, Kiebel SJ, Nichols TE, editors. Statistical parametric mapping: the analysis of functional brain images. Elsevier; 2011.

31. Calhoun VD, Wager TD, Krishnan A, Rosch KS, Seymour KE, Nebel MB, et al. The impact of T1 versus EPI spatial normalization templates for $\mathrm{FMRI}$ data analyses. Hum Brain Mapp. 2017;38:5331-42.

32. Greenwald AG, Nosek BA, Banaji MR. Understanding and using the Implicit Association Test: I. an improved scoring algorithm. J Pers Soc Psychol. 2003;85:197-216.

33. Kennerley SW, Walton ME. Decision making and reward in frontal cortex: Complementary evidence from neurophysiological and neuropsychological studies. Behav Neurosci. 2011;125:297-317.

34. Botvinick MM, Cohen JD, Carter CS. Conflict monitoring and anterior cingulate cortex: an update. Trends Cogn Sci. 2004;8:539-46.

35. Kolling N, Behrens TEJ, Mars RB, Rushworth MFS. Neural mechanisms of foraging. Science. 2012;335:95-98.

36. Boorman ED, Rushworth MF, Behrens TE. Ventromedial prefrontal and anterior cingulate cortex adopt choice and default reference frames during sequential multi-alternative choice. J Neurosci. 2013;33:2242-53.

37. Georgiadis JR, Kringelbach ML. The human sexual response cycle: brain imaging evidence linking sex to other pleasures. Prog Neurobiol. 2012;98:49-81.

38. Guillem K, Brenot V, Durand A, Ahmed SH. Neuronal representation of individual heroin choices in the orbitofrontal cortex. Addict Biol. 2018;23:880-8.

39. Schoenbaum G, Chiba AA, Gallagher M. Orbitofrontal cortex and basolateral amygdala encode expected outcomes during learning. Nat Neurosci. 1998;1:155-9.

40. Krueger CE, Laluz V, Rosen HJ, Neuhaus JM, Miller BL, Kramer JH. Double dissociation in the anatomy of socioemotional disinhibition and executive functioning in dementia. Neuropsychology. 2011;25:249.

41. Deng W, Rolls ET, Ji X, Robbins TW, Banaschewski T, Bokde ALW, et al. Separate neural systems for behavioral change and for emotional responses to failure during behavioral inhibition. Hum Brain Mapp. 2017;38:3527-37.

42. Voon V, Napier TC, Frank MJ, Sgambato-Faure V, Grace AA, Rodriguez-Oroz M, et al. Impulse control disorders and levodopa-induced dyskinesias in Parkinson's disease: an update. Lancet Neurol. 2017;16:238-50.

43. Politis M, Loane C, Wu K, O'Sullivan SS, Woodhead Z, Kiferle L, et al. Neural response to visual sexual cues in dopamine treatment-linked hypersexuality in Parkinson's disease. Brain. 2013;136:400-11.

44. Voon V, Mole TB, Banca P, Porter L, Morris L, Mitchell S, et al. Neural correlates of sexual cue reactivity in individuals with and without compulsive sexual behaviours. PLoS ONE. 2014;9.

45. Mechelmans DJ, Irvine M, Banca P, Porter L, Mitchell S, Mole TB, et al. Enhanced attentional bias towards sexually explicit cues in individuals with and without compulsive sexual behaviours. PLoS ONE. 2014;9:8.

46. Borsini F. KR Evans - US Patent 7 151,103, 2006 undefined. Method of treating female hypoactive sexual desire disorder with flibanserin. Google Patents. 2006.

47. Lenoir M, Serre F, Cantin L, Ahmed SH. Intense sweetness surpasses cocaine reward. PLoS ONE 2007;2:e698. 
The role of dopaminergic and serotonergic transmission in the processing... C Schmidt et al.

48. Mahler SV, Smith KS, Berridge KC. Endocannabinoid hedonic hotspot for sensory pleasure: anandamide in nucleus accumbens shell enhances 'liking' of a sweet reward. Neuropsychopharmacology. 2007;32:2267-78.

49. Cheer JF, Wassum KM, Heien MLAV, Phillips PEM, Wightman RM. Cannabinoids enhance subsecond dopamine release in the nucleus accumbens of awake rats. J Neurosci. 2004;24:4393-4400.

50. Peciña S, Cagniard B, Berridge KC, Aldridge JW, Zhuang X. Hyperdopaminergic mutant mice have higher 'wanting' but not 'liking' for sweet rewards. J Neurosci. 2003;23:9395-402.

51. McCabe C, Huber A, Harmer CJ, Cowen PJ. The D2 antagonist sulpiride modulates the neural processing of both rewarding and aversive stimuli in healthy volunteers. Psychopharmacology. 2011;217:271-8.

52. O'Doherty J, Rolls ET, Francis S, Bowtell R, McGlone F. Representation of pleasant and aversive taste in the human brain. J Neurophysiol. 2001;85:1315-21.

53. Knutson B, Adams CM, Fong GW, Hommer D. Anticipation of increasing monetary reward selectively recruits nucleus accumbens. J Neurosci. 2001;21:RC159.

54. Brand M, Snagowski J, Laier C, Maderwald S. Ventral striatum activity when watching preferred pornographic pictures is correlated with symptoms of Internet pornography addiction. Neuroimage. 2016;129:224-32.

55. Duvauchelle CL, Levitin M, MacConell LA, Lee LK, Ettenberg A. Opposite effects of prefrontal cortex and nucleus accumbens infusions of flupenthixol on stimulantinduced locomotion and brain stimulation reward. Brain Res. 1992:576:104-10.
56. Diekhof EK, Gruber O. When desire collides with reason: functional interactions between anteroventral prefrontal cortex and nucleus accumbens underlie the human ability to resist impulsive desires. J Neurosci. 2010;30:1488-93.

57. Grant JE, Kim SW, Potenza MN, Blanco C, Ibanez A, Stevens L, et al. Paroxetine treatment of pathological gambling: a multi-centre randomized controlled trial. Int Clin Psychopharmacol. 2003;18:243-9.

58. Gola M, Potenza MN. Paroxetine treatment of problematic pornography use: a case series. J Behav Addict. 2016;5:529-32.

59. DeRogatis LR, Komer L, Katz M, Moreau M, Kimura T, Garcia M Jr, et al. Treatment of hypoactive sexual desire disorder in premenopausal women: efficacy of flibanserin in the VIOLET Study. J Sex Med. 2012;9:1074-85.

60. Caruso S, Agnello C, Intelisano G, Farina M, Di Mari L, Cianci A. Placebo-controlled study on efficacy and safety of daily apomorphine SL intake in premenopausal women affected by hypoactive sexual desire disorder and sexual arousal disorder. Urology. 2004;63:955-9.

61. Masand PS, Ashton AK, Gupta S, Frank B. Sustained-release bupropion for selective serotonin reuptake inhibitor-induced sexual dysfunction: a randomized double-blind, placebo-controlled, parallel-group study. Am J Psychiatry. 2001;158:805-7.

62. Yee A, Loh HS, Ong TA, Ng CG, Sulaiman AH. Randomized, double-blind, parallelgroup, placebo-controlled trial of bupropion as treatment for methadoneemergent sexual dysfunction in men. Am J Mens Health. 2018;12:1705-18. 